\title{
Assessment of Global Fatigue in Multiple Sclerosis: A Spanish Language Version of the CGI and PGI Fatigue Scales
}

\author{
Steven D. Targum ${ }^{1}$, Pablo Richly ${ }^{2}$, Vladimiro Sinay ${ }^{2,3}$, Daniel Goldberg-Zimring ${ }^{1}$, Facundo Manes ${ }^{2}$ \\ ${ }^{1}$ Clintara LLC, Boston, USA; ${ }^{2}$ Institute of Neurosciences at Favaloro University, Buenos Aires, Argentina; ${ }^{3}$ Institute of Cognitive \\ Neurology (INECO), Buenos Aires, Argentina. \\ Email: sdtargum@clintara.com
}

Received May $13^{\text {th }}$, 2013; revised June $5^{\text {th }}$, 2013; accepted June $30^{\text {th }}, 2013$

Copyright (C) 2013 Steven D. Targum et al. This is an open access article distributed under the Creative Commons Attribution License, which permits unrestricted use, distribution, and reproduction in any medium, provided the original work is properly cited.

\begin{abstract}
Background: Fatigue is often identified as weakness following muscular exertion in patients with multiple sclerosis (MS) but may be associated with other physical, cognitive and emotional symptoms. Objective: To develop a Spanish language global impression of fatigue scales to evaluate symptoms of fatigue distinct from a particular disease. Methods: 50 ambulatory patients with MS attending a clinical institute in Argentina consented to participate in this reliability study. The Spanish language version of the Clinical and Patient Global Impressions of Fatigue (CGI-S-F and PGI-S-F) instruments were administered with the Massachusetts General Hospital cognitive and physical functioning questionnaire (MGH-CPFQ). Results: The CGI-S-F and PGI-S-F scores were well correlated with each other $(p<0.00005)$. The mean CGI-S for fatigue was $2.28 \pm 1.07$ (SD) and PGI-S for fatigue was $2.30 \pm 1.16$ ( $p=n s$ ) reflecting borderline to mild perception of fatigue. The total MGH-CPFQ was $16.68 \pm 4.32$. Both CGI-S-F and PGI-S-F measures were correlated with the MGH-CPFQ: CGI-Severity ( $r=0.632 ; p<0.00005)$; PGI-Severity $(r=0.717 ; p<0.00005)$. Conclusions: In this study, the Spanish language versions of the CGI-S-F and PGI-S-F were reliable measures in an MS population and can be useful and easily applied metrics in a busy clinical practice.
\end{abstract}

Keywords: Reliability; Multiple Sclerosis; Spanish; Global Assessment; Fatigue; Psychometrics; Treatment Response

\section{Introduction}

Approximately $50 \%$ to $60 \%$ of patients with Multiple Sclerosis (MS) describe fatigue as one of their most troubling symptoms, regardless of their disease course or level of disability [1]. Fatigue symptoms are often presented as part of the symptom cluster of MS and have been associated with regional cerebral brain atrophy in these patients [2-4]. In MS, fatigue is often experienced as weakness following muscular exertion (muscular fatigue) and may precede the other evolving symptoms of the disease [5]. In addition, Krupp and Elkins [6] found that following continuous effort. MS patients performed worse than control subjects on tests of visual and verbal memory reflecting the impact of mental fatigue. MS patients with symptoms of fatigue may have greater work and/or social performance difficulties and develop more health problems than less fatigued patients $[7,8]$. In the United States, the Social Security Administration recognizes fatigue as a significant cause of unemployment among people with MS [1].

The perception of fatigue may reflect different issues including muscular weakness, lassitude, daytime sleepiness, and/or the inability to focus [9-12]. Arnold [13] delineated three distinct categories of fatigue related to physical, cognitive, and emotional symptoms. The physical symptoms of fatigue include reduced activity, low energy, tiredness, decreased physical endurance, increased effort to do physical tasks, general weakness, heaviness, slowness or sluggishness, non-restorative sleep, and sleepiness. Clearly, many patients with MS experience these symptoms. However, additional cognitive and emotional symptoms may also be associated symptoms of fatigue. The cognitive symptoms include decreased concentration, decreased attention, decreased mental endurance, and slowed thinking. The emotional (affective) symptoms of fatigue include decreased motivation or initiative (apathy), decreased interest, feeling overwhelmed, feeling bored, aversion to effort, and feeling low. There- 
fore, the subjective experience and differential description of fatigue symptoms may differ markedly between patients.

In clinical practice, the evaluation and treatment of fatigue may be complicated because it is often part of the symptom cluster of MS, but can also be a symptom of another disorder distinct from MS, or a side effect of the medications used in the treatment of MS [10,11]. Furthermore, adding additional medications to treat the identified symptoms of fatigue adds risk because of the possibility of inducing additional adverse symptoms as a consequence of the intervention.

There are several instruments used to assess fatigue including the 9-item self-report fatigue severity scale (FSS), more comprehensive instruments examining vitality and inertia, and performance-based measures specifically related to MS [5,14-16]. The 11-item fatigue questionnaire has been used in clinical trials to assess symptoms of fatigue, and the fatigue descriptive scale (FDS) distinguishes fatigue at rest, during exercise, and worsening with exertion [15-17]. Alternatively, Schwid and colleagues [14] developed a quantitative, performancebased measure of motor fatigue using three exercise protocols to distinguish fatigue from weakness in individual muscles. Bakshi et al. [18] used the FSS and expanded disability status scale (EDSS) [19] to study fatigue and examine its relationship to depression and disability in 71 patients with multiple sclerosis. They reported that fatigue was significantly correlated with depression in these MS patients but was not associated with physical disability as measured by the EDSS.

Although useful in many instances, these aforementioned rating tools may be too time consuming for use in a busy clinic or limited in scope to either the clinician's or subjects' personal interpretation (e.g., FSS). In a busy clinical setting, it would be helpful to have a simpler, faster, yet reliable metric tool that can quickly assess both the clinician's and subject's independent assessment of fatigue symptoms. The Clinical Global Impression of severity scale (CGI-S) and related patient version (PGI-S) are easily understood, single score metrics that can be useful for this purpose [20,21]. Targum et al. [22] developed and validated a modified form of the CGI and PGI severity scales to specifically assess the severity of symptoms of fatigue in central nervous system (CNS) populations. This modified CGI severity scale for fatigue (CGI-S-F) provides specific "targeted" symptoms of fatigue to facilitate specific symptom identification and adds scoring anchors to improve the precision needed to assess these symptoms [23]. For this study, we translated these companion global assessment instruments into Spanish and assessed the utility and reliability of the Spanish-version CGI-S-F and PGI-S-F in an ambulatory clinical population of patients with MS.

\section{Methods and Materials}

\subsection{Study Description and Subject Population}

50 ambulatory patients with MS consented to participate in the assessment of a newly developed, Spanish-language global assessment scale for fatigue. All subjects were attending the Institute of Cognitive Neurology (INECO) located in Buenos Aires, Argentina, and were randomly selected to participate in the study between August 2011 and January 2012.

Clinicians administered the CGI-S-F scale to all subjects who independently completed the PGI-S-F and the Massachusetts General Hospital Cognitive and Physical Functioning Scale (MGH-CPFQ). The MGH-CPFQ is a 7-item patient-rated instrument that has been shown to be both valid and reliable in clinical trials [24].

\subsection{Description of the Instruments}

Both the CGI-S-F and PGI-S-F were designed as anchored instruments rated from 1 to 7 with increasing severity of fatigue based upon the last 7 days [22]. The descriptive anchors specifically focus on the identified symptoms of fatigue. For instance, a global severity score of 3 reflects mild fatigue whereas a severity of 4 reflects moderate fatigue. Both instruments include the same generic, yet "targeted" descriptors of possible symptoms that may be associated with fatigue in any medical condition, including MS.

For instance, the PGI-S descriptor refers to the individual (patient) completing the self-rating instrument and reads as follows in English:

Symptoms of fatigue may include effects on your physical wellbeing (such as low or decreased energy, tiredness, decreased physical endurance or ability to sustain physical activity, general weakness, heaviness in the arms or legs, general heaviness, slowness or sluggishness, sleepiness, increased effort with physical tasks) on your mood state (decreased motivation or interest, decreased effort or initiative), or your cognitive abilities (such as decreased concentration, decreased attention, slowed thinking, reduced mental sharpness).

The English versions of the CGI-S-F and PGI-S-F were translated into Spanish by one of the authors (FM) and cross-validated back into English prior to its use in the current study.

The MGH-CPFQ is a validated patient-rated scale scored from 1-6 with increasing severity that individually evaluates 7 distinct items: Motivation/Enthusiasm, Wakefulness/Alertness, Energy, Focus/Attention, Recall, Ability to find words, and Sharpness/Mental acuity.

\subsection{Statistical Analyses}

Statistical analyses included intra-class correlations, Pear- 
son's correlation coefficient, and paired t-test comparisons. The MGH-CPFQ was used to validate the PGI and CGI fatigue instruments.

\section{Results}

\subsection{Demographics}

50 subjects attending an outpatient clinic for multiple sclerosis consented to participate in this study. There were 12 men and 38 women. The mean age of the group was $41.6 \pm 11.6(\mathrm{SD})$ years.

\subsection{Reliability}

Both PGI-S and CGI-S were reliable measures of fatigue in this MS population. The mean scores for the PGI-S and CGI-S revealed borderline to mild fatigue although the scores ranged from 1 (normal) to 5 (marked fatigue). There were no statistically significant differences between the patient-rated and clinician-rated global measures of fatigue. The mean PGI-S score was $2.30 \pm 1.16$ (SD) and the mean CGI-S was $2.28 \pm 1.07(t=0.89 ; d f=$ $98 ; p=n s)$. The intra-class correlation between the clinician-rated CGI-S and patient-rated PGI-S was $r=0.9465$ $(p<0.0001)$.

\subsection{Validation of the Global Impressions Instrument}

The MGH-CPFQ was included in this study to assess the validity of the CGI-S and PGI-S for fatigue. The mean MGH-CPFQ score was $16.68 \pm 4.48$. Most subjects perceived mild cognitive or physical symptoms although the scores ranged from 1 to 5 in this ambulatory population. Most of these MS subjects did not equate the experience of fatigue related to their illness with any impairment of motivation, alertness, energy, focus, or their cognitive abilities.

The Pearson's correlation with the total MGH-CPFQ score was 0.6316 for the PGI-S and 0.7173 for the CGI-S. Both CGI-S and PGI-S scores were highly correlated with the total MGH-CPFQ $(\mathrm{p}<0.001)$ and each of the individual 7 items.

Table 1 reveals the mean CGI-S scores and the Pearson's correlation for each of the individual CPFQ items.

\section{Discussion}

In this study, a Spanish language version of a validated global assessment instrument (the PGI and CGI for severity of fatigue) was both reliable and valid when administered to a population of ambulatory patients with MS. Each of the seven items of the MGH-CPFQ was highly correlated with the CGI-S and PGI-S for fatigue reflecting the validity of the instrument.
Table 1. Pearson Correlation: CGI-S Fatigue with individual MGH-CPFQ items in an MS population $(n=50)$.

\begin{tabular}{cccc}
\hline Comparison & $\begin{array}{c}\text { MGH-CPFQ } \\
\text { Score } \\
\text { (mean } \pm \text { SD) }\end{array}$ & $\begin{array}{c}\text { Pearson’s } \\
\text { correlation }\end{array}$ & $p$-value \\
\hline CGI-S to Motivation & $2.40 \pm 1.07$ & 0.560 & $<0.005$ \\
CGI-S to Wakefulness & $2.40 \pm 0.86$ & 0.521 & $<0.005$ \\
CGI-S to Energy & $2.76 \pm 1.04$ & 0.501 & $<0.005$ \\
CGI-S to Focus & $2.36 \pm 0.88$ & 0.609 & $<0.005$ \\
CGI-S to Recall & $2.44 \pm 0.86$ & 0.528 & $<0.005$ \\
CGI-S to Ability & $2.22 \pm 0.58$ & 0.456 & $<0.005$ \\
CGI-S to Sharpness & $2.10 \pm 0.58$ & 0.480 & $<0.005$ \\
\hline
\end{tabular}

Fatigue symptoms often present as part of the symptom cluster of MS as weakness following muscular exertion and/or cognitive deficits [5,6]. However, in this study the majority of MS subject's perceived their global fatigue as only borderline or mild severity (mean PGI-S score of 2.30). These global scores were essentially equivalent to the scores obtained from a healthy, comparison group (mean PGI-S $=2.39$ ), and substantially lower than the scores for subjects with psychiatric disorders (mean PGI-S score $=3.92$ ) examined in the United States [22]. The MGH-CPFQ scores submitted by the MS patients were also similar to the healthy controls in the US study [22]. Therefore, these MS subjects did not equate the fatigue often related to their illness with impairment of motivation, energy, focus, or with their cognitive abilities. Although this finding may be due to cultural or geographical differences related to fatigue perception, it is more likely due to the specific, relatively stable patients making routine visits to this specific ambulatory clinic and the broad diversity of fatigue symptom severity observed in MS patients. The extremely high correlation noted between the PGI-S and CGI-S scores $(r=0.9465)$ suggests that the patient perceptions are at least consistent with the independent clinical judgment of the clinician.

Fatigue symptoms can impact productivity, interpersonal relations, and the sense of well being across the CNS spectrum including patients with depression, multiple sclerosis, and schizophrenia [5,7-9,12,13].

Our objectives of this study were to develop a Spanish language version of the validated CGI fatigue scale and apply it in a different CNS population. A limitation of this study is that we do not compare our CGI fatigue instrument with other commonly used, but longer fatigue instruments, like the FSS or the revised 11-item fatigue questionnaire [5,17]. In addition, we did not include the EDSS [19] as an independent measure of disabling distinct from fatigue. Our intent was not to replace these 
tools but to design a simple, reliable single item metric that would be applicable for both clinicians and patients. The PGI-S and CGI-S instruments that we have developed do provide a single item, overall impression that assesses three distinct categories of fatigue related to physical, cognitive, and emotional symptoms. Both the English and Spanish language versions offer detailed descriptors of these three categories to facilitate symptom identification and customized scoring anchors to facilitate accurate scoring.

We have now employed the CGI fatigue instrument in American psychiatric patients (Major Depressive Disorder and schizophrenia) and healthy controls, and in a second study of Latin patients with multiple sclerosis. In each group, there was a high correlation between the CGI-S and PGI-S as well as high correlations with the MGH-CPFQ providing a validation of the instrument. In both studies, the CGI-S and PGI-S were easily understood and administered by both the clinic staff and consenting subjects. Consequently, we believe this instrument can be a meaningful metric when applied in a busy clinic setting to reliably gauge symptoms of fatigue distinct from the specific CNS disorder being treated.

\section{REFERENCES}

[1] O. Hadjimichael, T. Vollmer and M. Oleen-Burkey, "Fatigue Characteristics in Multiple Sclerosis: The North American Research Committee on Multiple Sclerosis (NARCOMS) Survey," Health and Quality of Life Outcomes, Vol. 6, 2008, pp. 100-110. doi:10.1186/1477-7525-6-100

[2] R. A. Marrie, E. Fisher, D. M. Miller, J. Lee and R. A. Richard, "Association of Fatigue and Brain Atrophy in Multiple Sclerosis,” Journal of the Neurological Sciences, Vol. 228, No. 2, 2005, pp. 161-166. doi:10.1016/j.jns.2004.11.046

[3] G. Tedeschi, D. Dinacci, L. Lavorgna, et al., "Correlation between Fatigue and Brain Atrophy and Lesion Load in Multiple Sclerosis Patients Independent of Disability," Journal of the Neurological Sciences, Vol. 263, No. 1, 2007, pp. 15-19. doi:10.1016/j.jns.2007.07.004

[4] A. K. Andreasen, J. Jakobsen, L. Soerensen, et al., "Regional Brain Atrophy in Primary Fatigued Patients with Multiple Sclerosis,” Neuroimage, Vol. 50, No. 2, 2010, pp. 608-615. doi:10.1016/j.neuroimage.2009.12.118

[5] L. B. Krupp, N. G. LaRocca, J. Muir-Nash and A. D. Steinberg, "The Fatigue Severity Scale, Application to Patients with Multiple Sclerosis and Systemic Lupus Erythematosus," Archives of Neurology, Vol. 46, No. 10, 1989, pp. 1121-1123.

doi:10.1001/archneur.1989.00520460115022

[6] L. B. Krupp and L. E. Elkins, "Fatigue and Declines in Cognitive Functioning in Multiple Sclerosis,” Neurology, Vol. 55, No. 7, 2000, pp. 934-939.

doi:10.1212/WNL.55.7.934

[7] S. Sevy, M. H. Rosenthal, J. Alvir, et al., "Double-Blind,
Placebo-Controlled Study of Modafinil for Fatigue and Cognition in Schizophrenia Patients Treated with Psychotropic Medications," Journal of Clinical Psychiatry, Vol. 66, 2005, pp. 839-843. doi:10.4088/JCP.v66n0705

[8] R. S. McIntyre, J. Z. Konarksi, J. K. Soczynska, et al., "Medical Comorbidity in Bipolar Disorder: Implications for Functional Outcomes and Health Service Utilization," Psychiatric Services, Vol. 57, No. 8, 2006, pp. 1140-1144. doi:10.1176/appi.ps.57.8.1140

[9] E. A. Walker, W. J. Katon and R. P. Jemelka, "Psychiatric Disorders and Medical Care Utilization among People in the General Population Who Report Fatigue,” Journal of General Internal Medicine, Vol. 8, No. 8, 1993, pp. 436-440. doi:10.1007/BF02599621

[10] M. Fava, "Symptoms of Fatigue and Cognitive/Executive Dysfunction in Major Depressive Disorder before and after Antidepressant Treatment," Journal of Clinical Psychiatry, Vol. 64, No. 14, 2003, pp. 30-34.

[11] K. Demyttenaere, J. Fruyt and S. M. Stahl, "The Many Faces of Fatigue in Major Depressive Disorder,” International Journal of Neuropharmacology, Vol. 8, No. 1, 2005, pp. 93-105.

[12] S. B. Harvey, S. Wessely, D. Kuh and M. Hotopf, “The Relationship between Fatigue and Psychiatric Disorders: Evidence for the Concept of Neurasthenia," Journal of Psychosomatic Research, Vol. 66, No. 5, 2009, pp. 445454. doi:10.1016/j.jpsychores.2008.12.007

[13] L. M. Arnold, "Understanding Fatigue in Major Depressive Disorder and Other Medical Disorders,” Psychosomatics, Vol. 49, No. 3, 2008, pp. 185-190. doi:10.1176/appi.psy.49.3.185

[14] S. R. Schwid, C. A. Thornton, S. Pandya, et al., "Quantitative Assessment of Motor Fatigue and Strength in MS," Neurology, Vol. 53, No. 4, 1999, pp. 743-750. doi:10.1212/WNL.53.4.743

[15] C. Christopoulou, "The Assessment and Measurement of Fatigue,” In: J. DeLuca, Ed., Fatigue as a Window to the Brain, MIT Press, New York, 2005, pp. 19-35.

[16] L. Whitehead, "The Measurement of Fatigue in Chronic Illness: A Systematic Review of Unidimensional and Multidimensional Fatigue Measures," Journal of Pain and Symptom Management, Vol. 37, No. 1, 2009, pp. 107-128. doi:10.1016/j.jpainsymman.2007.08.019

[17] P. Ferrentinos, V. Kontaxakis, B. Havaki-Kontaxaki, D. Dikeos and G. Papadimitriou, "The Fatigue Questionnaire: Standardization in Patients with Major Depression,” Psychiatry Research, Vol. 177, No. 1, 2010, pp. 114-119. doi:10.1016/j.psychres.2009.01.029

[18] R. Bakshi, Z. A. Shaikh, R. S. Miletich, et al., "Fatigue in Multiple Sclerosis and Its Relationship to Depression and Neurologic Disability,” Multiple Sclerosis, Vol. 6, No. 3, 2000, pp. 181-185.

[19] J. F. Kurtzke, "Rating Neurologic Impairment in Multiple Sclerosis: An Expanded Disability Status Scale (EDSS)," Neurology, Vol. 33, No. 11, 1983, pp. 1444-1452. doi:10.1212/WNL.33.11.1444

[20] W. Guy, "ECDEU Assessment Manual for Psychopharmacology,” DHEW Publication ADM-76-338, 1976. 
[21] J. Busner and S. D. Targum, "The Clinical Global Impressions Scale: Applying a Research Tool in Clinical Practice,” Psychiatry, Vol. 4, No. 7, 2007, pp. 28-37.

[22] S. D. Targum, H. Hassman, M. Pinho and M. Fava, “Development of a Clinical Global Impression Scale for Fatigue,” Journal of Psychosomatic Research, Vol. 46, No. 3, 2012, pp. 370-374.

doi:10.1016/j.jpsychires.2011.12.001

[23] S. D. Targum, J. Busner and A. H. Young, "Targeted
Scoring Criteria Reduce Variance in Global Impressions,” Human Psychopharmacology: Clinical and Experimental, Vol. 23, No. 7, 2008, pp. 629-633. doi:10.1002/hup.966

[24] M. Fava, D. V. Iosifescu, P. Pedrelli and L. Baer, "Reliability and Validity of the Massachusetts General Hospital Cognitive and Physical Functioning Questionnaire," Psychotherapy and Psychosomatics, Vol. 78, 2009, pp. 91-97. doi:10.1159/000201934 